\title{
Comprehensive peptide quantification for data independent acquisition mass spectrometry using chromatogram libraries
}

\author{
Brian C. Searle ${ }^{1,2}$, Lindsay K. Pino ${ }^{1}$, Jarrett D. Egertson ${ }^{1}$, Ying S. \\ Ting $^{1}$, Robert T. Lawrence ${ }^{1}$, Judit Villén ${ }^{1}$, and Michael J. MacCoss ${ }^{1 *}$ \\ 1Department of Genome Sciences, University of Washington, Seattle, WA, USA \\ 2Proteome Software, Portland, OR, USA \\ *Corresponding author, email: maccoss@uw.edu
}

\begin{abstract}
Data independent acquisition (DIA) mass spectrometry is a powerful technique that is improving the reproducibility and throughput of proteomics studies. We introduce a new experimental workflow that uses this technique to construct chromatogram libraries that capture fragment ion chromatographic peak shape and retention time for every detectable peptide in an experiment. These coordinates calibrate information in spectrum libraries or protein databases to a specific mass spectrometer and chromatography setup, and enable sensitive peptide detection in quantitative experiments. We also present EncyclopeDIA, a software tool for generating and searching chromatogram libraries, and demonstrate the performance of our workflow by quantifying proteins in human and yeast cells. We find that by exploiting calibrated retention time and fragmentation specificity in chromatogram libraries, EncyclopeDIA can detect and quantify $>50 \%$ more peptides from DIA experiments than with DDAbased spectrum libraries alone.
\end{abstract}

\section{INTRODUCTION}

Over the past two decades the continued refinement of proteomics methods using liquid chromatography (LC) coupled to tandem mass spectrometry (MS/MS) has enabled a deeper understanding of human biology and disease $(1,2)$. Recently data independent acquisition( 3,4$)(\mathrm{DIA})$, in which the mass spectrometer systematically 
acquires MS/MS spectra irrespective of whether or not a precursor signal is detected, has emerged as a powerful alternative approach to data dependent acquisition(5) (DDA) for proteomics experiments. In current DIA workflows, instrument cycle is structured such that the same MS/MS spectrum window is collected every 1 to 5 seconds, enabling quantitative measurements using fragment ions instead of precursor ions. This approach produces data analogous to targeted parallel reaction monitoring (PRM), except instead of targeting specific peptides, quantitative data is acquired across a predefined mass to charge $(\mathrm{m} / \mathrm{z})$ range. One trade-off is that to cover the $\mathrm{m} / \mathrm{z}$ space where the majority of peptides exist, the mass spectrometer must be tuned to produce MS/MS spectra with wide precursor isolation windows that often contain multiple peptides at the same time. These additional peptides produce interfering fragment ions, and database search engines for DDA that rely on a precursor isolation window of at most a few daltons can struggle to detect the signal for a particular peptide from that background interference. The PAcIFIC approach(6) attempts to overcome this difficulty by using multiple gas-phase fractionated injections of the same sample to increase precursor isolation at the cost of both sample and instrument time.

Peptide-centric tools analyze DIA measurements for individual peptides across all spectra in a precursor isolation window. Spectrum library search tools for DIA data(7-9) use fragmentation patterns and relative retention times from previously collected DDA data. In contrast, other tools such as PECAN(10) query DIA data using just peptide sequences and their predicted fragmentation pattern without requiring a spectrum library. While library searching can achieve better sensitivity than PECAN, the approach is limited to detecting only analytes represented in the library. In addition, the quality of library-based detections is only as strong as the quality of the library itself. Because mapping fragmentation patterns and retention times across instruments and platforms is difficult, many researchers prefer to simultaneously acquire both DDA and DIA data from their samples $(11,12)$. While this implicitly increases the acquisition time and sample consumption, it becomes possible to detect peptides using the DDA data while making peptide quantitation measurements using the DIA data. However, detection sensitivity is inherently limited to that of the DDA data. 
Typically tens to hundreds of biological samples are processed and analyzed using LC-MS/MS in quantitative proteomics experiments. In DDA workflows each individual sample is informatically processed alone to account for stochastic variation in data acquisition. The regularity of DIA allows researchers to make peptide detections in one sample and transfer those detections to other samples(13). Here we extrapolate this concept by collecting certain runs where data acquisition is tuned to improve peptide detection rates, while collecting other runs with a focus on quantification accuracy and throughput. Results from runs dedicated to peptide detection are formed into a DIA-based chromatogram library. In a chromatogram library, we catalog retention time, precursor mass, peptide fragmentation patterns, and known interferences that identify each peptide on our instrumentation within a specific sample matrix.

We have developed EncyclopeDIA, a library search engine that takes full advantage of chromatogram libraries, and we demonstrate a substantial gain in sensitivity over typical DIA and DDA workflows. EncyclopeDIA also contains several new approaches to automate transition refinement to remove fragment ion interference, improving the quality of quantification. This tool is instrument vendor neutral and available as an open source project with both a GUI and command line interface.

\section{RESULTS}

\section{Chromatogram library generation.}

Chromatogram libraries differ from spectrum libraries in that they are generated from a small collection of narrow-window DIA experiments, rather than from DDA. We use a data acquisition scheme (Figure 1a) similar to PAcIFIC(6) for constructing chromatogram libraries. Briefly, for each experiment we create a representative sample, which pools subaliquots of each biological sample. We acquire six or more gas-phase fractionated runs from this pooled sample in an effort to comprehensively study all of the peptides in the pool. The gas-phase fractionated samples are injected into the mass spectrometer multiple times, with each injection staggered to acquire a fraction of the 
$\mathrm{m} / \mathrm{z}$ space via overlapping $4 \mathrm{~m} / \mathrm{z}$ "narrow" precursor isolation windows. After overlap deconvolution, these experiments effectively have $2 \mathrm{~m} / \mathrm{z}$ precursor isolation (analogous to if we had conducted targeted PRM acquisition) except we are targeting all precursors between 400 to $1000 \mathrm{~m} / \mathrm{z}$. Previously we have shown that this type of DIA experiment can produce substantially richer peptide detection lists than similarly acquired DDA experiments(10). While this data acquisition strategy would be impractical to perform for every biological sample, when applied to the pool it provides the mass, retention time, and fragmentation coordinates for virtually every detectable peptide in the experiment, which we use to lookup the peptides in quantitative samples.

\section{The EncyclopeDIA workflow.}

EncyclopeDIA is comprised of several algorithms for DIA data analysis (Figure 1b) that can search for peptides using either DDA-based spectrum libraries or DIAbased chromatogram libraries. The algorithms in this workflow are described in full detail in the Online Methods. Briefly, the EncyclopeDIA workflow starts with reading raw MS/MS data in mzML files into an SQLite database designed for querying fragment spectra across precursor isolation windows. If fragment spectra are collected using overlapping windows, they are deconvoluted on the fly during file reading. Libraries are read as DLIB (DDA-based spectrum libraries) or ELIB (EncyclopeDIA DIA-based chromatogram libraries). EncyclopeDIA determines the highest scoring retention time point corresponding to each library spectrum (as well as a paired reverse sequence decoy) using a scoring system modeled after the X!Tandem HyperScore(14). Fifteen auxiliary match features (not based on retention time) are calculated at this time point. These features are aggregated and submitted to Percolator 3.1(15), a semi-supervised SVM algorithm for interpreting target/decoy peptide detections, for a first pass validation. EncyclopeDIA generates a retention time model from peptides detected at 1\% FDR using a non-parametric kernel density estimation algorithm that follows the density mode across time. Any target or decoy peptide in the feature set that does not match the retention time model is reconsidered up to 5 times until we find a highest 
scoring retention time point that matches the model. The retention time-curated feature sets are submitted to Percolator for final pass validation at $1 \%$ FDR.

\section{Comparison between spectrum libraries and chromatogram libraries.}

EncyclopeDIA can be used to query DIA data with DDA-based spectrum libraries. However, the benefit of the algorithms in EncyclopeDIA become transparent when using DIA-based chromatogram libraries. EncyclopeDIA can generate ELIB chromatogram libraries from gas-phase fractionated runs using DDA-based spectrum libraries if they are available, or using Walnut, which is a built-in, performance optimized re-implementation of the PECAN algorithm(10) to search protein sequence FASTA databases (see Supplementary Note 1 for further details). The resulting ELIB report can be fed back into EncyclopeDIA for chromatogram library searching. While this approach is inherently limited to the detectable proteins in the narrow-window pool, our perspective is that except for rare variants, very few quantitatively reliable peptides will be detectable in the wide-window data that are not also detectable in the narrow data. In cases where rare variants are important to a study or if samples are likely to represent very disparate proteomes, EncyclopeDIA can also generate chromatogram libraries from multiple batches of narrow-window acquisitions from different sample pools.

We evaluated the chromatogram library strategy using peptides derived from a HeLa S3 cell lysate as a representative high-complexity proteome. To this end we constructed a chromatogram library from six gas-phase fractionated DIA runs with 52 overlapping $4 \mathrm{~m} / \mathrm{z}$-wide windows, which produced $3002 \mathrm{~m} / \mathrm{z}$-wide windows spanning 400.43 to $1000.70 \mathrm{~m} / \mathrm{z}$ after deconvolution. Following the scheme in Figure 1a, we searched the narrow-window data against a HeLa-specific DDA spectrum library containing $166.4 \mathrm{k}$ unique peptides. This produced a chromatogram library containing 99.6k unique peptides where the retention times, fragmentation patterns, and interference likelihoods were calibrated to our mass spectrometer and HPLC setup. We performed an analogous approach using Walnut to detect peptides directly from the 
narrow-window DIA data using a Uniprot Human FASTA database, which generated a 53.2k peptide chromatogram library. The performance separation between these two library-generation methods is in part because the spectrum library represents a more targeted search space.

In addition to generating the library, we also collected triplicate wide-window DIA runs with 52 overlapping $24 \mathrm{~m} / \mathrm{z}$-wide windows from the same sample. From these runs we were able to detect an average of $20.6 \mathrm{k}$ peptides from the Uniprot Human FASTA database using Walnut. In contrast, we found an average of $47.8 \mathrm{k}$ peptides $(2.3 \mathrm{x}$ increase) when we searched the Walnut-based chromatogram library with EncyclopeDIA (Figure 2a). Requiring only an additional 6 injections, this search strategy found nearly an equal number of peptides compared to searching the SCX-fractionated, 36 injection DDA-based spectrum library (an average of $48.7 \mathrm{k}$ peptides). Finally, we found an average of $72.3 \mathrm{k}$ peptides when searching against the chromatogram library constructed using the DDA-based spectrum library. Here we detected over 2x more peptides than our benchmark top-20 DDA experiments (Supplementary Figure 1). Despite this increased detection rate, we still find that DIA produces more consistent results compared to DDA, as indicated by the overlap in peptide detections between triplicate injections (Figure $2 \mathrm{~b}$ and $2 \mathrm{c}$ ).

Confirming these results, we performed the same analysis using a yeast cell lysate and found similar improvement rates when comparing Walnut versus EncyclopeDIA using a Walnut-based chromatogram library (2.2x increase, Figure 2d). Here we observe more modest gains over top-20 DDA experiments, which likely reflects the lowered proteomic complexity of yeast versus human cells and is echoed in the tight overlap (86\%) between triplicate DIA injections versus DDA (Figure $2 e$ and $2 f)$. As is possible with any computational strategy that incorporates machine learning, we were concerned with the potential for overfitting that might manifest in over exaggerated peptide detection rates. To answer this question we searched the HeLa wide-window DIA data using the yeast chromatogram library (and vice versa) to verify that we see a negative result when searching the wrong library. As expected this result (Figure 2a and $2 \mathrm{~d}$ ) produced zero peptide detections that passed a $1 \%$ peptide FDR threshold. 
We also find that DIA analysis with chromatogram libraries is more sensitive at detecting low abundance proteins at a 1\% protein FDR. Using tandem affinity purification tagging and quantitative Western blots, Ghaemmaghami et al(16) quantified 3868 yeast proteins with more than 50 estimated copies per cell. In this study we replicated strain and growing conditions as closely as possible to use their measurements as an independent benchmark. While both DDA and DIA confidently detect the majority of proteins at levels above $10^{4}$ copies per cell, DIA outperforms DDA by $49 \%$ with proteins estimated to have between $10^{3}$ and $10^{4}$ copies per cell and by $2 x$ with proteins estimated between $10^{2}$ and $10^{3}$ copies per cell (Figure 3 ).

\section{Improved retention time and fragmentation pattern calibration in chromatogram}

\section{libraries.}

One of the primary reasons on-column chromatogram libraries enable such high performance is that they exploit within run retention time reproducibility. Accurate retention time filtering is an important consideration when analyzing high-complexity proteomes with DIA, and virtually all DIA library search engines make use of this data. Retention times in aggregate spectrum libraries are typically derived by linearly interpolating multiple DDA data sets to a known calibration space (such as that defined by the iRT standard (17)), which enables retention times to be comparable from run to run, or even across platforms. However, these measurements usually contain some wobble due to errors introduced by assuming a linear fit. Figure $4 a$ shows a typical spread of retention times in EncyclopeDIA detected peptides using a DDA spectrum library, which is $95 \%$ accurate within a spread of 5.1 minutes (Figure 4c). In comparison, Figure $4 \mathrm{~b}$ shows the typical spread of retention times in the chromatogram library, which is 95\% accurate within 21 seconds (Figure 4d). This tightening of retention time accuracy is due to the fact that chromatogram libraries are collected on the same column as the wide-window acquisitions. Even if efforts are made to keep packing material, length, and gradient consistent, the dramatic gains in retention time accuracy with chromatogram libraries reflect variations that are difficult to control for, including 
packing speeds, pressures, and pulled tip orifice shapes. In addition, we find that DDA fragmentation patterns (Figure 4e) are often somewhat different than those collected in DIA experiments (Figure 4f). While DDA instrument methods usually tune MS/MS collision energies to the precursor charge and mass, some of this variation is likely due to fixed assumptions in charge states and precursor masses required by DIA methods when multiple precursors must be fragmented at the same time.

A subtle issue with DIA library searching when using generalized spectrum libraries is that many peptides generate the same fragment ions, either because of sequence variation, paralogs, or modified forms. While EncyclopeDIA attempts to control for this using background ion distributions to predict interference likelihoods, sequence variation due to homology or single nucleotide polymorphisms can be unintentionally detected as the wrong peptide sequence in certain circumstances. For example, a sequence variation of a valine to an isoleucine is relatively common, and the mass shift of a methyl group $(+14 / Z)$ will often place both peptides inside the same precursor isolation window when $Z$ is 2 or greater. Using chromatogram libraries can provide some protection against these issues because the initial searches to generate the libraries are performed using narrow $(2 \mathrm{~m} / \mathrm{z}$ ) precursor mass windows, and subsequent wide-window searches benefit from precise retention time filtering. Additionally, EncyclopeDIA requires at least $25 \%$ of the primary score to come from ions that indicate the modified form to detect modified peptides when modified/unmodified peptide pairs fall in the same precursor isolation window (e.g. methionine oxidation).

\section{Peptide and protein quantitation.}

We present a novel algorithm for automated transition refinement to remove fragment ion interference and alleviate the need for manual curation (see Online Methods for further details). In short, after unit area normalizing all transitions assigned to a single peptide (Supplementary Figure 2a), we determine the shape of the peak as the median normalized intensity at each retention time point (Supplementary Figure $2 b$ ). 
Transitions that match this peak shape with Pearson's correlation scores $>0.9$ are considered quantitative (Supplementary Figure 2c). We find that over $81 \%$ of peptides can be quantified with at least three transitions (Supplementary Figure 3a) and that the transitions picked by our approach produce reproducible quantitative measurements between technical replicates in HeLa experiments (Supplementary Figure 3b and 3c).

Combining peptide detections across multiple samples often increases false discoveries because false detections are usually found only in individual runs(18). To combat this, we recalculate global peptide FDR across all experiments in each study with Percolator and generate parsimonious protein detection lists that are also filtered to a $1 \%$ FDR. We use cross-sample retention time alignment(13) to help quantify peptides that are missing in specific samples. After filtering peptides based on coefficient of variance and measurement consistency we estimate protein quantities by summing fragment ion intensities across only sequence-unique peptides assigned to those proteins.

\section{Determining global proteomic changes from serum starvation.}

We used the chromatogram library approach to examine the effects of serum starvation in human cells. Serum starvation is a common step in signal transduction studies as serum contains several cytokines and growth factors that can confound signaling levels. It is commonly thought that serum starvation suppresses basal activity by reducing signaling activity that effectively resets cells to G0/G1 resting phase(19), although more recent experiments $(20,21)$ suggest otherwise. Serum starvation protocols vary widely from 2 to 24 hours, and this time frame is long enough to produce changes in protein levels resulting from transcriptional regulation. These changes are a source of variation that can have serious consequences when comparing between studies.

We designed a DIA quantitative experiment to map how the proteome of HeLa cells changes in response to serum starvation over time. We selected starvation times 
to match commonly used protocols. Of the 99.6k unique peptides in our chromatogram library, we recapitulated 93.5k unique peptides from 6,802 protein groups in at least one quantitative sample at a global protein FDR $<0.01$. Of these, $48.6 \mathrm{k}$ peptides (from 5,781 protein groups) produced at least three quantitative transition ions without interference, had $<20 \%$ study-wide CVs, and were measured in every replicate of at least one time point. While at first these detection and quantification criteria may seem unusually stringent compared to typical proteomics experiments, narrowing our focus to confident measurements increased power in detecting subtle quantitative differences with high accuracy.

We found that 1097 protein groups in the HeLa proteome changed significantly over time at an FDR of 0.01 (Supplementary Table 1). The temporal starvation profiles of these proteins fell into five groups (Figure 5) where the majority changing proteins increased in abundance. Several of these proteins are involved in expected pathways such as cell cycle regulation (GO enrichment FDR=0.011), metabolism (GO enrichment $F D R=0.011$ ), and ubiquitination regulation ( $G$ enrichment FDR=0.018). One advantage of our method is that quantitation is performed by summing peaks from several low interference fragment ions, which allows us to accurately quantify small changes. For example, we found that all eight of the observed components of the nuclear proteasome increased significantly by approximately $25 \%$ (Supplementary Figure 4), which indicates nuclear maintenance consistent with G0/G1 resting phase.

We also observed significant regulation of the abundance of 39 kinases and 7 phosphatases (Supplementary Figure 5). In particular, we found that EGFR levels increased by $30 \%$ over a 24 hour serum starvation time course (Supplementary Figure 6), effectively sensitizing HeLa to the growth factor EGF. To confirm these experiments, we monitored relative changes in the phosphoproteome of HeLa after EGF stimulation at two common serum starvation times: 4 hours and 16 hours. We found that while phosphopeptide measurements at both time points directionally agreed, some phosphopeptide responses to EGF were stronger when cells were starved for 16 hours compared to when starving for only 4 hours (Supplementary Figure 7). This increase corroborated our observation that EGFR protein levels increased from 4 to 16 hours of 
starvation. These protein and phosphopeptide-level changes underline a potentially significant source of variation when comparing phosphorylation signaling studies.

\section{DISCUSSION}

We have demonstrated an experimental strategy that enables comprehensive detection of peptides and proteins using chromatogram libraries. These libraries can be seeded either with a DDA spectrum library or generated in a DIA-only mode using Walnut for initial peptide searches. Finally, we showed that at the cost of only six additional narrow-window DIA runs, both of these strategies are more sensitive and reproducible relative to comparable DDA experiments. While this approach may be unrealistic for one-off experiments, we feel that in most quantitative proteomics studies the addition of these runs are a minor cost in exchange for a significant increase in sensitivity.

One important limitation of our method is that each chromatogram library is tuned for a specific mass spectrometer and chromatographic set up. In particular, we have observed that with the hand-pulled and packed columns used here, there is significant retention time variation between replicates run on different columns, even if effort is made to insure column consistency. We hypothesize that minor variations in packing speeds, packing pressures, tip shapes, and column lengths can affect elution times and even peptide retention time ordering. This issue may be mitigated by acquiring a new library after a column change and retention time aligning the libraries to insure consistency. Future work remains to model these minor retention time shifts.

Another important consideration is library quality. All library searching strategies assume that entries in the library are correctly identified and consequently false positives in the library can be propagated as "true" positives by target/decoy analysis(22). This concern is potentially compounded in our approach, which can include up to two levels of library creation. Further work is necessary to improve FDR estimates for library searching in DIA experiments. In the meantime, we feel orthogonal 
filtering strategies are necessary to maintain conservative peptide detection lists. In addition to retention time fitting and $1 \%$ protein-level FDR filtering, in this work we require a minimum of three interference-free transitions and impose stringent measurement reproducibility requirements for peptides to be considered quantitative.

We have observed a complementarity of DDA and DIA through the use of building spectrum libraries to seed chromatogram libraries. Here the stochasticity of DDA sampling when coupled with offline peptide separation methods such as SCX fractionation can be exploited as a benefit in that only one observation of a peptide is necessary for inclusion in the library. With human samples, libraries constructed using previously recorded retention times and fragmentation patterns contained nearly twice the peptides as those constructed without prior knowledge. However, PECAN/Walnut can build on that knowledge by detecting peptide sequence variants illuminated by whole exome sequencing(10), and we are exploring ways of generating chromatogram libraries that incorporate both pieces of data.

\section{ACKNOWLEDGEMENTS}

We would like to thank members of the Villén and MacCoss labs for critical discussions. We additionally thank N. Shulman for implementing Skyline visualization of EncyclopeDIA reports, and S. Just, P. Seitzer, and S. Ludwigsen for EncyclopeDIA bug reports and patches. B.C.S. is supported by F31 GM119273; L.K.P. is supported by F31 AG055257. This work is supported by P41 GM103533, R21 CA192983, and U54 HG008097 to M.J.M.; and R35 GM119536, R01 AG056359, and a research grant from the W.M. Keck Foundation to J.V.

\section{AUTHOR CONTRIBUTIONS}

B.C.S. and M.J.M. conceived the study. B.C.S., R.T.L., and M.J.M designed the 
experiments. B.C.S., L.K.P., and R.T.L. performed the experiments. B.C.S. designed and wrote the software with input from L.K.P., J.D.E., and Y.S.T.. BCS analyzed the data. M.J.M. and J.V. supervised the work. B.C.S., L.K.P., J.D.E., Y.S.T., R.T.L., J.V., and M.J.M. wrote the paper.

\section{COMPETING FINANCIAL INTERESTS}

The MacCoss Lab at the University of Washington has a sponsored research agreement with Thermo Fisher Scientific, the manufacturer of the instrumentation used in this research. Additionally, M.J.M. is a paid consultant for Thermo Fisher Scientific. 


\section{REFERENCES}

1. Mertins $\mathrm{P}$, Mani DR, Ruggles $\mathrm{KV}$ et al. Proteogenomics connects somatic mutations to signalling in breast cancer. Nature. 2016;534:55-62.

2. Zhang B, Wang J, Wang $X$ et al. Proteogenomic characterization of human colon and rectal cancer. Nature. 2014;513:382-387.

3. Venable JD, Dong MQ, Wohlschlegel J, Dillin A, Yates JR. Automated approach for quantitative analysis of complex peptide mixtures from tandem mass spectra. Nat Methods. 2004;1:39-45.

4. Gillet LC, Navarro P, Tate $S$ et al. Targeted data extraction of the MS/MS spectra generated by data-independent acquisition: a new concept for consistent and accurate proteome analysis. Mol Cell Proteomics. 2012;11:0111.016717.

5. Stahl DC, Swiderek KM, Davis MT, Lee TD. Data-controlled automation of liquid chromatography/tandem mass spectrometry analysis of peptide mixtures. J Am Soc Mass Spectrom. 1996;7:532-540.

6. Panchaud A, Scherl A, Shaffer SA et al. Precursor acquisition independent from ion count: how to dive deeper into the proteomics ocean. Anal Chem. 2009;81:6481-6488.

7. Weisbrod CR, Eng JK, Hoopmann MR, Baker T, Bruce JE. Accurate peptide fragment mass analysis: multiplexed peptide identification and quantification. $\mathrm{J}$ Proteome Res. 2012;11:1621-1632.

8. Röst HL, Rosenberger G, Navarro P et al. OpenSWATH enables automated, targeted analysis of data-independent acquisition MS data.[letter]. Nat Biotechnol 2014;32(3):219-223.

9. Bruderer R, Bernhardt OM, Gandhi T et al. Extending the limits of quantitative proteome profiling with data-independent acquisition and application to acetaminophen-treated three-dimensional liver microtissues. Mol Cell Proteomics. 2015;14:1400-1410.

10. Ting YS, Egertson JD, Bollinger JG et al. PECAN: library-free peptide detection for data-independent acquisition tandem mass spectrometry data. Nat Methods. 2017; 14:903-908.

11. Egertson JD, MacLean B, Johnson R, Xuan Y, MacCoss MJ. Multiplexed peptide analysis using data-independent acquisition and Skyline. Nat Protoc. 2015;10:887903. 
12. Schubert OT, Gillet LC, Collins BC et al. Building high-quality assay libraries for targeted analysis of SWATH MS data. Nat Protoc. 2015;10:426-441.

13. Röst HL, Liu Y, D’Agostino G et al. TRIC: an automated alignment strategy for reproducible protein quantification in targeted proteomics. Nat Methods. 2016;13:777-783.

14. Fenyö D, Beavis RC. A method for assessing the statistical significance of mass spectrometry-based protein identifications using general scoring schemes. Anal Chem. 2003;75:768-774.

15. The M, MacCoss MJ, Noble WS, Käll L. Fast and Accurate Protein False Discovery Rates on Large-Scale Proteomics Data Sets with Percolator 3.0. J Am Soc Mass Spectrom. 2016;27:1719-1727.

16. Ghaemmaghami S, Huh WK, Bower K et al. Global analysis of protein expression in yeast. Nature. 2003;425:737-741.

17. Escher C, Reiter L, MacLean B et al. Using iRT, a normalized retention time for more targeted measurement of peptides. Proteomics. 2012;12:1111-1121.

18. Rosenberger G, Bludau I, Schmitt U et al. Statistical control of peptide and protein error rates in large-scale targeted data-independent acquisition analyses. Nat Methods. 2017;14:921-927.

19. Pardee AB. G1 events and regulation of cell proliferation. Science. 1989;246:603608.

20. Levin VA, Panchabhai SC, Shen L, Kornblau SM, Qiu Y, Baggerly KA. Different changes in protein and phosphoprotein levels result from serum starvation of highgrade glioma and adenocarcinoma cell lines. J Proteome Res. 2010;9:179-191.

21. Pirkmajer S, Chibalin AV. Serum starvation: caveat emptor. Am J Physiol Cell Physiol. 2011;301:C272-9.

22. Lam H, Deutsch EW, Eddes JS et al. Development and validation of a spectral library searching method for peptide identification from MS/MS. Proteomics. 2007;7:655-667. 


\section{Figure 1}

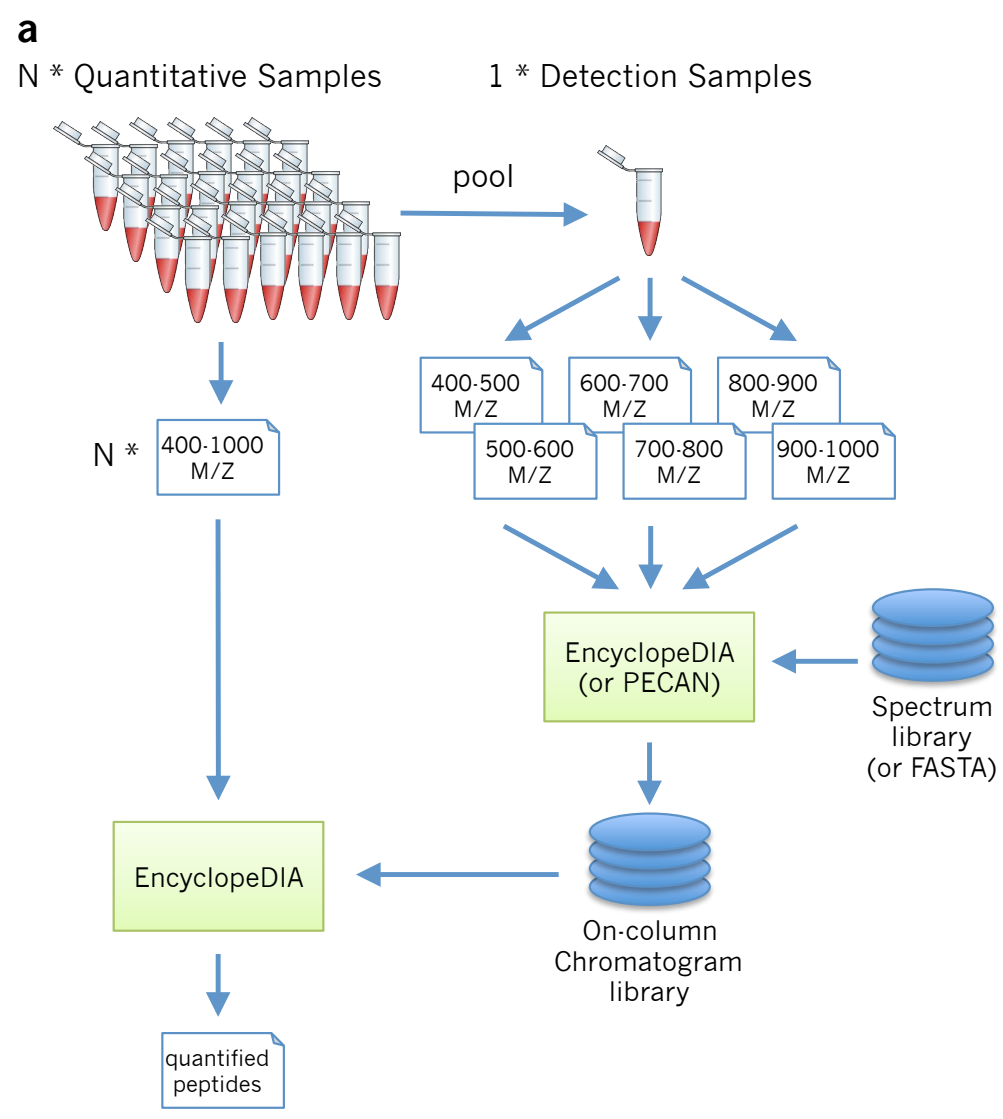

b

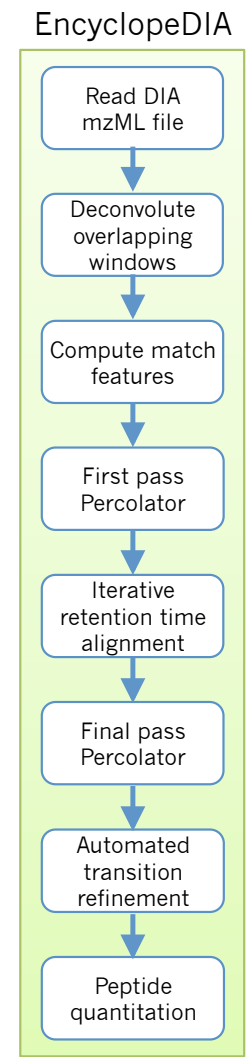

An approach for quantifying peptides with chromatogram libraries. (a) The chromatogram library generation workflow. Briefly, in addition to collecting wide-window DIA experiments on each quantitative replicate, a pool containing peptides from every condition is measured using several staggered narrow-window DIA experiments. After deconvolution, these narrow-window experiments have $2 \mathrm{~m} / \mathrm{z}$ precursor isolation, which is analogous to targeted parallel reaction monitoring (PRM) experiments, except effectively targeting every peptide between 400 and $1000 \mathrm{~m} / \mathrm{z}$. We detect peptide anchors from these experiments using either EncyclopeDIA (searching a DDA spectrum library) or PECAN (using a protein database) and chromatographic data about each peptide is stored in a chromatogram library with retention times, peak shape, fragment ion intensities, and known interferences tuned specifically for the LC/MS/MS setup. EncyclopeDIA then uses these precise coordinates for $\mathrm{m} / \mathrm{z}$, time, and intensity to detect peptides in the quantitative samples. (b) The EncyclopeDIA algorithmic workflow for searching spectrum and chromatogram libraries. After reading and deconvoluting DIA raw files, EncyclopeDIA calculates several retention time independent feature scores for each peptide that are amalgamated and FDR corrected with Percolator. Using high confidence peptide detections, EncyclopeDIA retention time warps detections to the library, determines the retention time accuracy, and reconsiders outliers. After a second FDR correction with Percolator, EncyclopeDIA autonomously picks fragment ion transitions that fit each non-parametrically calculated peak shape and quantifies peptides using these ions. 


\section{Figure 2}

a

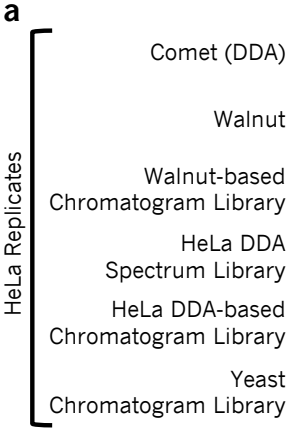

d

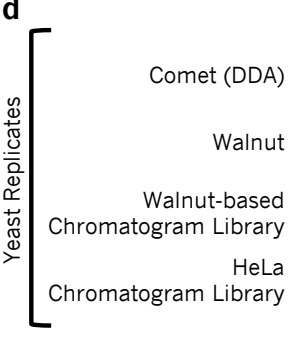

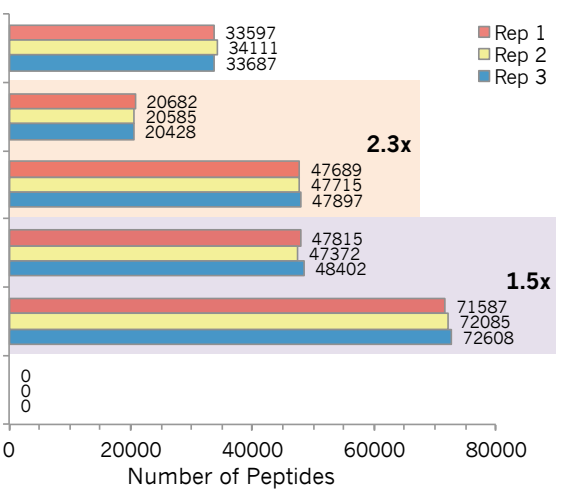

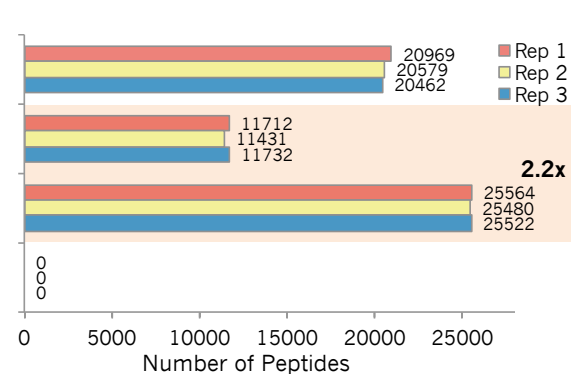

b

Comet (DDA)

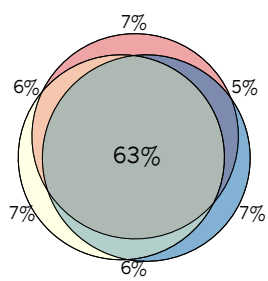

e

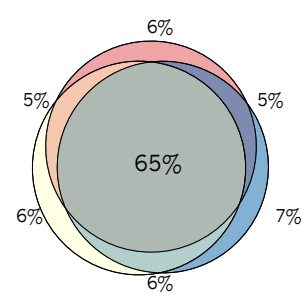

C

HeLa DDA-based Chromatogram Library

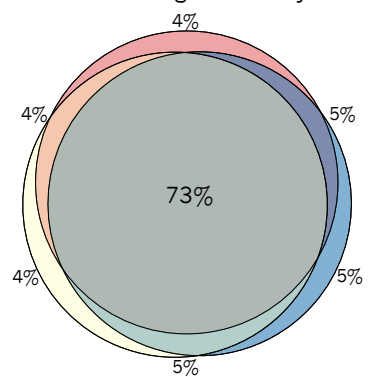

f

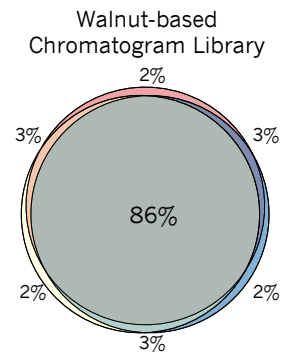

Untargeted peptide detection rates using DDA and DIA from human and yeast cell lysates. We used EncyclopeDIA to search chromatogram and spectrum libraries, while we used Comet and Walnut to search DDA and DIA data directly using FASTA protein databases. (a) The number of peptide detections at $1 \%$ peptide FDR in triplicate HeLa injections. (b) The overlap in HeLa S3 peptide detections between replicates using DDA searched by Comet and (c) using DIA searched by EncyclopeDIA where the size of Venn diagram circles in HeLa analyses are consistent with the number of detections. (d) The number of peptide detections at $1 \%$ peptide FDR in triplicate BY4741 yeast injections. (e) The overlap in yeast peptide detections between replicates using DDA searched by Comet and (f) using DIA searched by EncyclopeDIA where the size of circles are consistent with the number of yeast peptide detections. 


\section{Figure 3}
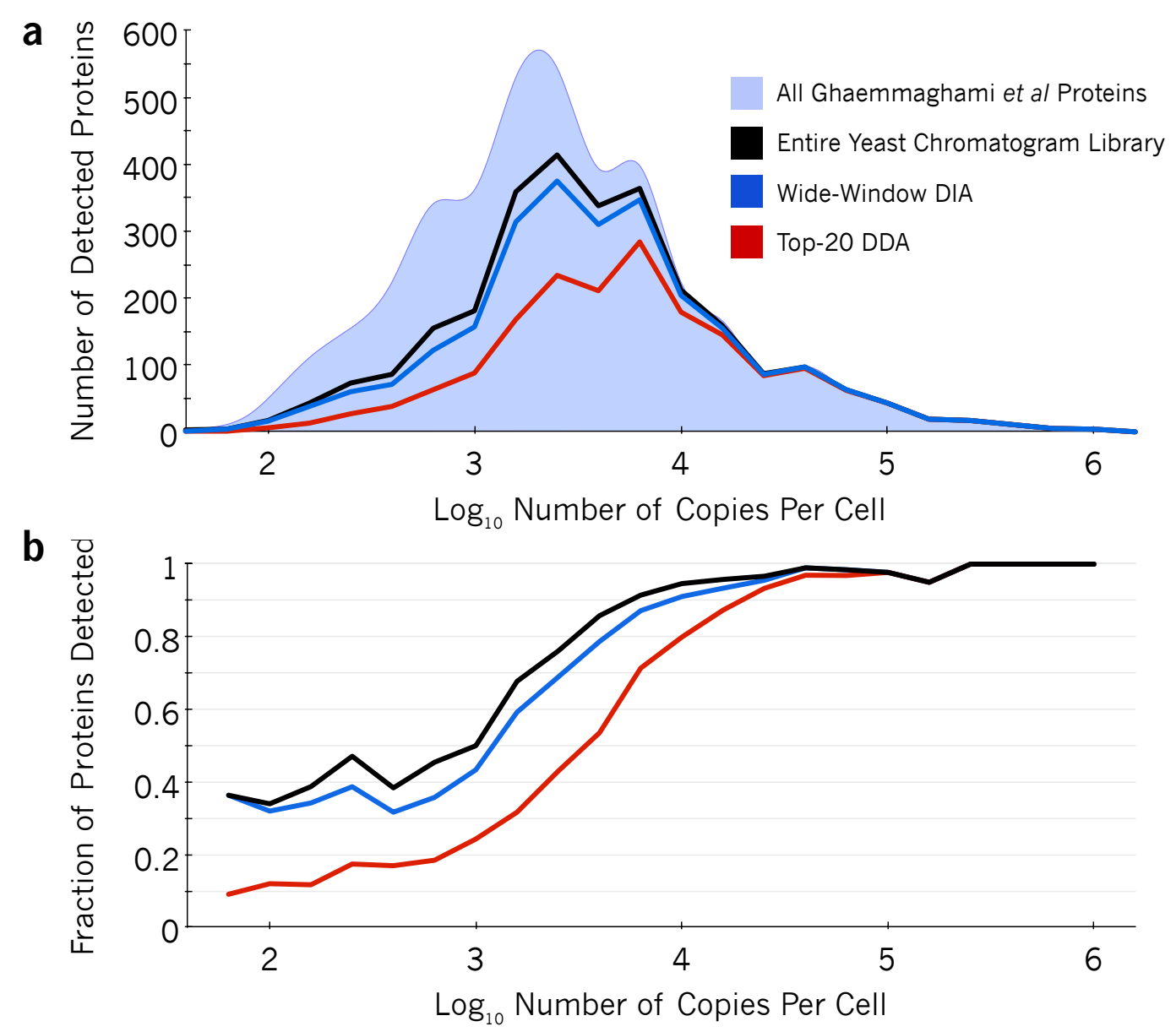

Protein detection rates scale with abundance. The (a) number and (b) fraction of proteins detected in yeast at different orders of magnitude of abundance.

Ghaemmaghami et al comprehensively estimated protein copies per cell in yeast (light blue area) using high-affinity epitope tagging. While top-20 DDA (red line) can measure some low abundant proteins at $1 \%$ protein-level FDR, the strategy only detected $48 \%$ of mid-range proteins with estimated copies per cell between $10^{3}$ and $10^{4}$. In contrast, at $1 \%$ protein-level FDR, wide-window DIA using a Walnut-based chromatogram library (blue line) detected $71 \%$ of these proteins and overall recapitulated $91 \%$ of proteins found in the entire Walnut-based chromatogram library (black line). 


\section{Figure 4}
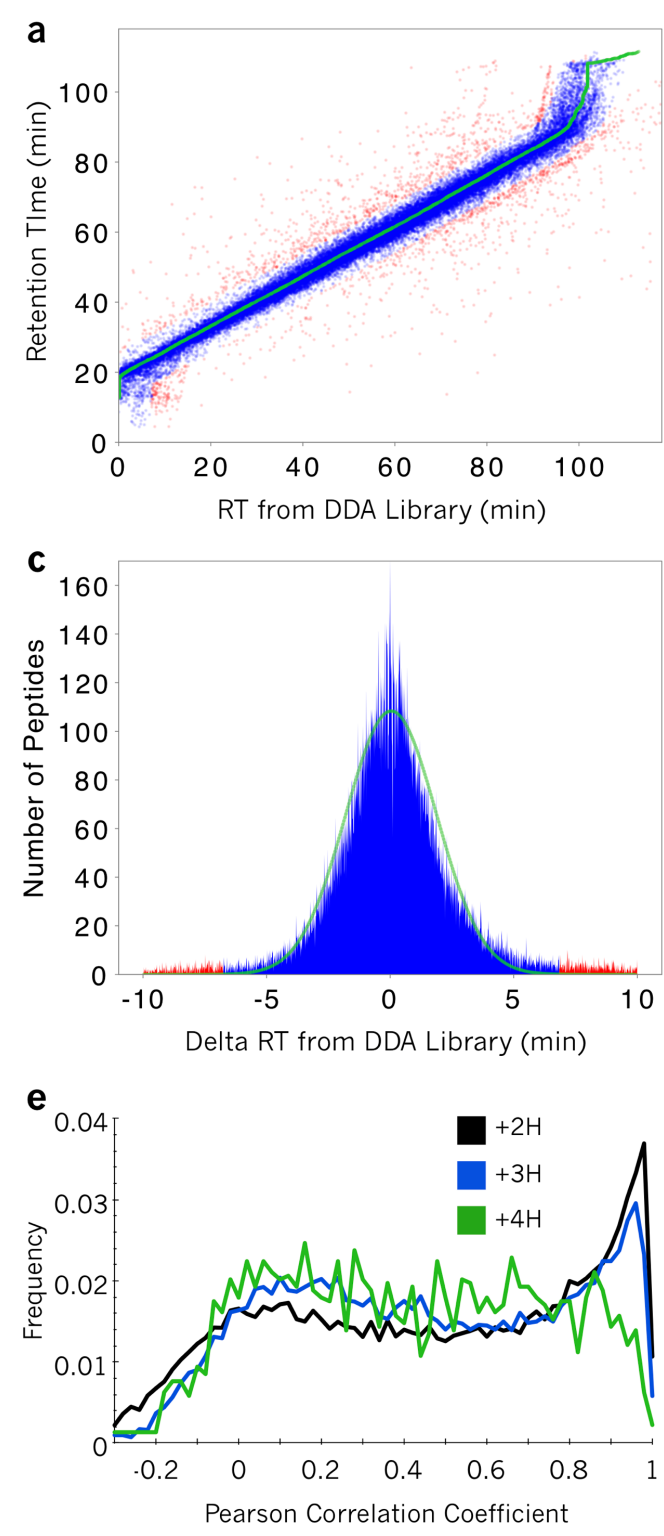
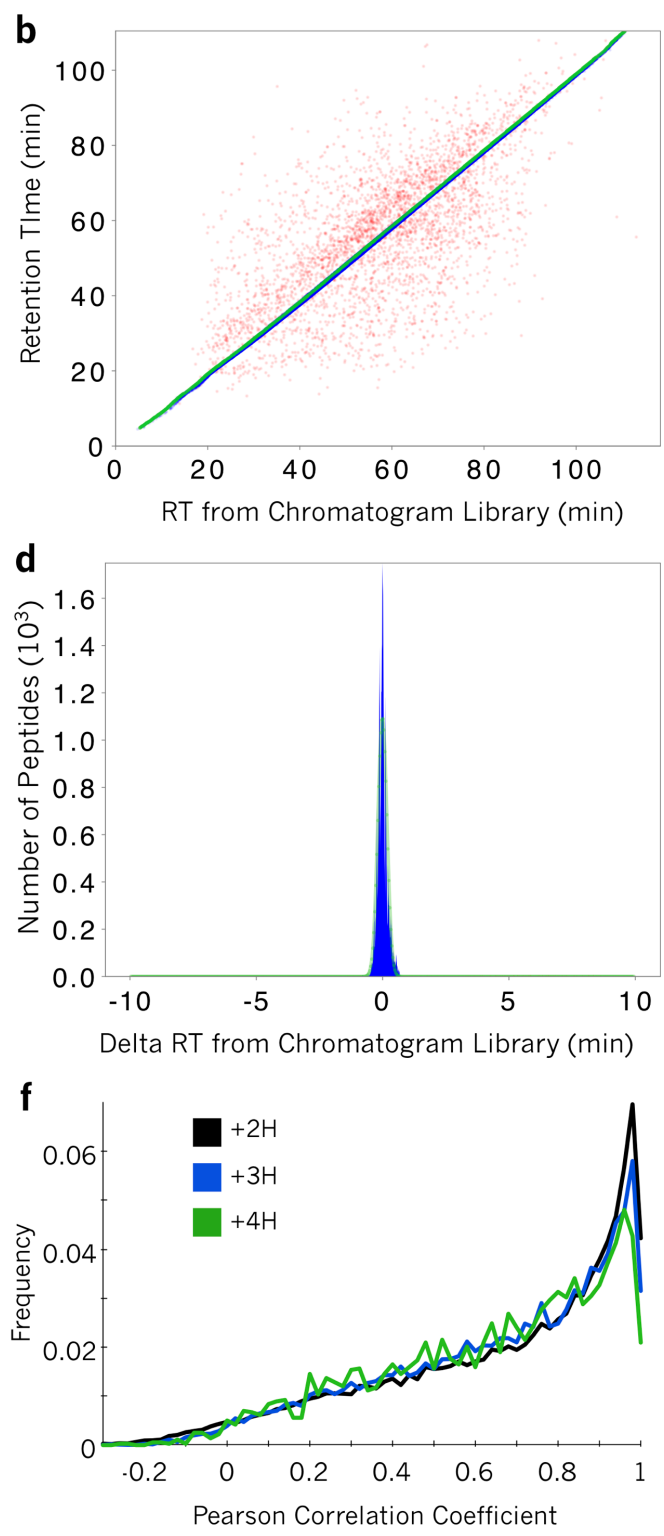

Retention time and fragmentation accuracy of the DDA spectrum library and the DIA chromatogram library. Scatterplots comparing retention times from the (a) DDA spectrum library and the (b) DIA chromatogram library to those from in a single HeLa DIA experiment. Each point represents a peptide, where blue peptides fit the retention time trend (green) within a Bayesian mixture model probability of $5 \%$ and red peptides are outliers (see Online Methods for more details). (c) Retention times in the DDA spectrum library are $95 \%$ accurate to a window of 5.1 minutes, while (d) retention times in the chromatogram library are $95 \%$ accurate to 21 seconds. (e) The distribution of Pearson correlation coefficients between spectra in the DDA spectrum library and those detected from a single HeLa DIA experiment shows charge state bias, while (f) the distribution of correlation coefficients between spectra in the DIA chromatogram library and those from the same experiment shows much less bias. 


\section{Figure 5}

a

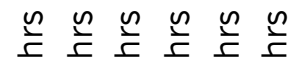

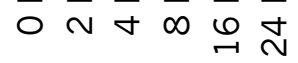

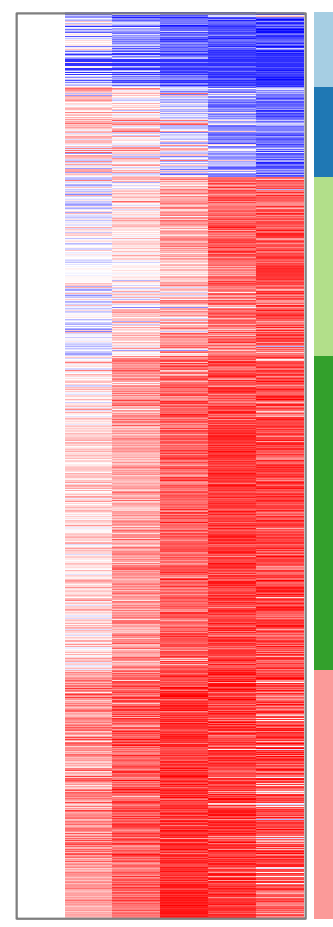

-3 Z-score +3 b
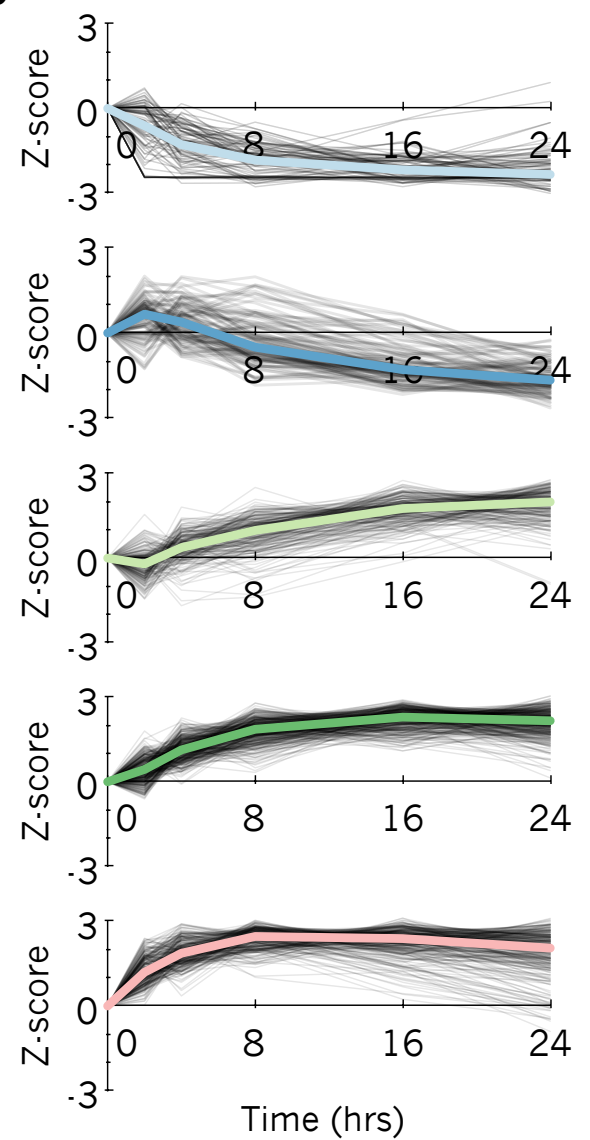

Protein quantification changes following serum starvation. (a) Heatmap of 1097 proteins found to be quantitatively changing at a FDR corrected p-value $<0.01$ in HeLa. Colors are Z-score normalized and indicate the number of standard deviations away from the level at time 0. (b) Protein changes grouped into five K-means clusters (see Supplementary Figure 12 for more details) showing separation between fast responding proteins (light blue, dark green, and pink) and delayed responses (dark blue, light green). 


\section{METHODS}

HeLa cell culture and sample preparation. HeLa S3 cervical cancer cells (ATCC) were cultured at $37^{\circ} \mathrm{C}$ and $5 \% \mathrm{CO}_{2}$ in Dulbecco's modified Eagle's medium (DMEM) supplemented with L-glutamine, $10 \%$ fetal bovine serum (FBS), and $0.5 \%$ strep/penicillin. Six cell culture replicates were grown to approximately a $50 \%$ density in 6-well plates prior to FBS starvation staggered for 24, 16, 8, 4, 2, and 0 hours (one time point in each well, one plate per replicate). At the 0 hour time point cells were quickly washed three times with refrigerated phosphate-buffered saline and immediately flash frozen with liquid nitrogen. Frozen cells were lysed in a buffer of $9 \mathrm{M}$ urea, $50 \mathrm{mM}$ Tris (pH 8), $75 \mathrm{mM} \mathrm{NaCl}$, and a cocktail of protease inhibitors (Roche Complete-mini EDTAfree). After scraping, cells were subjected to $2 \times 30$ seconds of probe sonication, 20 minutes of incubation on ice, followed by 10 minutes of centrifugation at $21,000 \times g$ and $4^{\circ} \mathrm{C}$. The protein content of the supernatant was estimated using BCA. The proteins were reduced with $5 \mathrm{mM}$ dithiothreitol for 30 minutes at $55^{\circ} \mathrm{C}$, alkylated with $10 \mathrm{mM}$ iodoacetamide in the dark for 30 minutes at room temperature, and quenched with an additional $5 \mathrm{mM}$ dithiothreitol for 15 minutes at room temperature. The proteins were diluted to $1.8 \mathrm{M}$ urea and then digested with sequencing grade trypsin (Pierce) at a 1:50 enzyme to substrate ratio for 12 hours at $37^{\circ} \mathrm{C}$. The digestion was quenched by adding $10 \%$ trifluoroacetic acid to achieve approximately $\mathrm{pH} 2$. Resulting peptides were desalted with $100 \mathrm{mg}$ tC18 SepPak cartridges (Waters) using vendor-provided protocols and dried with vacuum centrifugation. Peptides were brought to $1 \mu \mathrm{g} / 3 \mu \mathrm{l}$ in $0.1 \%$ formic acid (buffer A) prior to mass spectrometry acquisition. For the reproducibility experiments and to build a chromatogram library we pooled aliquots from all six time points for three of the replicates to ensure that the pool contained virtually every peptide present in the individual time points.

With the phosphoproteomics experiment, four replicates were performed for each of the four conditions: 20 minute EGF $(100 \mathrm{ng} / \mathrm{ml})$ or phosphate-buffered saline (PBS) stimulation following 4 hour starvation, and 20 minute EGF/PBS stimulation following 16 hour starvation. Sample generation and processing was performed in the same fashion with the following exceptions: 1 ) in addition to protease inhibitors, a cocktail of 
phosphatase inhibitors (50 mM NaF, $50 \mathrm{mM} \beta$-glycerophosphate, $10 \mathrm{mM}$ pyrophosphate, and $1 \mathrm{mM}$ orthovanadate) was also added to the lysis buffer, 2) proteins were digested for 14 hours, and 3) phosphopeptides were enriched using immobilized metal affinity chromatography (IMAC) using Fe-NTA magnetic agarose beads (Cube Biotech). Enrichment was performed with a KingFisher Flex robot (Thermo Scientific), which incubated peptides with $150 \mu \mathrm{l} 5 \%$ bead slurry in $80 \%$ acetonitrile, $0.1 \%$ TFA for 30 minutes, washed them three times with the same solution, and eluted them with 60 $\mu \mathrm{l} 50 \%$ acetonitrile: $1 \% \mathrm{NH}_{4} \mathrm{OH}$. Phosphopeptides were then acidified with $10 \%$ formic acid and dried. Phosphopeptides were brought to $1 \mu \mathrm{g} / 3 \mu \mathrm{lin} 0.1 \%$ formic acid assuming a 1:100 reduction in peptide abundance from the IMAC enrichment. Again, to build a chromatogram library we pooled aliquots from all four conditions for three of the replicates to ensure that the pool contained virtually every peptide present in the individual conditions.

Yeast cell culture and sample preparation. Yeast strain BY4741 (Dharmacon) was cultured at $30^{\circ} \mathrm{C}$ in YEPD and harvested at mid-log phase. Cell pellets were lysed in a buffer of $8 \mathrm{M}$ urea, $50 \mathrm{mM}$ Tris ( $\mathrm{pH} 8), 75 \mathrm{mM} \mathrm{NaCl}, 1 \mathrm{mM}$ EDTA (pH 8) using 7 cycles of 4 minutes bead beating with glass beads followed by one minute rest on ice. Lysate was collected by piercing the tube, placing it into an empty eppendorf, and centrifuging for 1 minute at $2000 \mathrm{rpm}$ and 4C. Insoluble material was removed from the lysate by 15 min centrifugation at $14000 \mathrm{rpm}$ and 4C. The protein content of the supernatant was estimated using BCA. The proteins were reduced with $5 \mathrm{mM}$ dithiothreitol for 30 minutes at $55^{\circ} \mathrm{C}$ and alkylated with $10 \mathrm{mM}$ iodoacetamide in the dark for 30 minutes at room temperature. The proteins were diluted to $1.8 \mathrm{M}$ urea and then digested with sequencing grade trypsin (Pierce) at a 1:50 enzyme to substrate ratio for 16 hours at $37^{\circ} \mathrm{C}$. The digestion was quenched using $5 \mathrm{~N} \mathrm{HCl}$ to achieve approximately $\mathrm{pH} 2$. Resulting peptides were desalted with $30 \mathrm{mg} \mathrm{MCX}$ cartridges (Waters) and dried with vacuum centrifugation. Peptides were brought to $1 \mu \mathrm{g} / 3 \mu \mathrm{l}$ in $0.1 \%$ formic acid (buffer A) prior to mass spectrometry acquisition.

Liquid chromatography mass spectrometry. Peptides were separated with a Waters NanoAcquity UPLC and emitted into a Thermo Q-Exactive HF or a Thermo Fusion 
tandem mass spectrometer. Pulled tip columns were created from $75 \mu \mathrm{m}$ inner diameter fused silica capillary in-house using a laser pulling device and packed with $3 \mu \mathrm{m}$ ReproSil-Pur C18 beads (Dr. Maisch) to $300 \mathrm{~mm}$. Trap columns were created from 150 $\mu \mathrm{m}$ inner diameter fused silica capillary fritted with Kasil on one end and packed with the same $\mathrm{C} 18$ beads to $25 \mathrm{~mm}$. Solvent $A$ was $0.1 \%$ formic acid in water, while solvent $B$ was $0.1 \%$ formic acid in $98 \%$ acetonitrile. For each injection, $3 \mu \mathrm{l}$ (approximately $1 \mu \mathrm{g}$ ) was loaded and eluted using a 90 -minute gradient from 5 to $35 \%$ B, followed by a 40 minute washing gradient. Data were acquired using either data-dependent acquisition (DDA) or data-independent acquisition (DIA). Three DDA and DIA HeLa and yeast technical replicates were acquired by alternating between acquisition modes to minimize bias. Serum-starved HeLa acquisition was randomized within blocks to enable downstream statistical analysis.

DDA acquisition and processing. The Thermo Q-Exactive HF was set to positive mode in a top-20 configuration. Precursor spectra $(400-1600 \mathrm{~m} / \mathrm{z})$ were collected at 60,000 resolution to hit an AGC target of $3 \mathrm{e} 6$. The maximum inject time was set to 100 ms. Fragment spectra were collected at 15,000 resolution to hit an AGC target of 1e5 with a maximum inject time of $25 \mathrm{~ms}$. The isolation width was set to $1.6 \mathrm{~m} / \mathrm{z}$ with a normalized collision energy of 27 . Only precursors charged between +2 and +4 that achieved a minimum AGC of 5 e3 were acquired. Dynamic exclusion was set to "auto" and to exclude all isotopes in a cluster. Thermo RAW files were converted to $\mathrm{mzXML}$ format using ReAdW and searched against a Uniprot Human FASTA database (87613 entries) with Comet (version 2015.02v2), allowing for variable methionine oxidation, and n-terminal acetylation. Cysteines were assumed to be fully carbamidomethylated. Searches were performed using a 50 ppm precursor tolerance and a 0.02 Da fragment tolerance using fully tryptic specificity $(\mathrm{KR} \mid \mathrm{P})$ permitting up to two missed cleavages. Search results were filtered to a $1 \%$ peptide-level FDR using Percolator (version 3.1).

DIA acquisition and processing. For each chromatogram library, the Thermo QExactive HF was configured to acquire six chromatogram library acquisitions with $4 \mathrm{~m} / \mathrm{z}$ DIA spectra (4 m/z precursor isolation windows at 30,000 resolution, AGC target 1e6, maximum inject time $55 \mathrm{~ms}$ ) using an overlapping window pattern from narrow mass 
ranges using window placements optimized by Skyline (i.e. 396.43 to $502.48 \mathrm{~m} / \mathrm{z}$, 496.48 to $602.52 \mathrm{~m} / \mathrm{z}, 596.52$ to $702.57 \mathrm{~m} / \mathrm{z}, 696.57$ to $802.61 \mathrm{~m} / \mathrm{z}, 796.61$ to 902.66 $\mathrm{m} / \mathrm{z}$, and 896.66 to $1002.70 \mathrm{~m} / \mathrm{z}$ ). See Supplementary Figure 8 and Supplementary Table 2 for the actual windowing scheme. Two precursor spectra, a wide spectrum $(400-1600 \mathrm{~m} / \mathrm{z}$ at 60,000 resolution) and a narrow spectrum matching the range (i.e. $390-510 \mathrm{~m} / \mathrm{z}, 490-610 \mathrm{~m} / \mathrm{z}, 590-710 \mathrm{~m} / \mathrm{z}, 690-810 \mathrm{~m} / \mathrm{z}, 790-910 \mathrm{~m} / \mathrm{z}$, and 890-1010 m/z) using an AGC target of $3 \mathrm{e} 6$ and a maximum inject time of 100 ms were interspersed every $18 \mathrm{MS} / \mathrm{MS}$ spectra.

For quantitative samples, the Thermo Q-Exactive HF was configured to acquire $25 \times 24 \mathrm{~m} / \mathrm{z}$ DIA spectra (24 m/z precursor isolation windows at 30,000 resolution, AGC target 1e6, maximum inject time $55 \mathrm{~ms}$ ) using an overlapping window pattern from 388.43 to $1012.70 \mathrm{~m} / \mathrm{z}$ using window placements optimized by Skyline. See Supplementary Figure 9 and Supplementary Table 2 for the actual windowing scheme. Precursor spectra (385-1015 m/z at 30,000 resolution, AGC target 3e6, maximum inject time $100 \mathrm{~ms}$ ) were interspersed every 10 MS/MS spectra. Phosphopeptide samples were analyzed in the same way using $20 \times 20 \mathrm{~m} / \mathrm{z}$ DIA spectra in an overlapping window pattern from 490.47 to $910.66 \mathrm{~m} / \mathrm{z}$.

All DIA spectra were programed with a normalized collision energy of 27 and an assumed charge state of +2 . Thermo RAW files were converted to.$m z M L$ format using the ProteoWizard package (version 3.0.7303) where they were peak picked using vendor libraries. A HeLa-specific Bibliospec(1) HCD spectrum library was created from unpublished Thermo Q-Exactive DDA data using Skyline (version 3.1.0.7382). This BLIB library and accompanying iRTDB normalized retention time database were converted into a ELIB library and used to search the mzMLs for peptides. EncyclopeDIA searches DIA data using $+1 \mathrm{H}$ and $+2 \mathrm{H}$ b/y ion fragments that could be found in library spectra. EncyclopeDIA was configured with default settings (10 ppm precursor, fragment, and library tolerances, considering both $B$ and $Y$ ions, and trypsin digestion was assumed). EncyclopeDIA was configured to use Percolator version 3.1. Phosphopeptides were processed the same way except a HeLa-specific phosphopeptide HCD spectrum library was used(2) and phosphopeptides detected in 
EncyclopeDIA searches were localized using Thesaurus (Searle et al in review).

Overlapping DIA deconvolution. When using the overlapping DIA scheme, every spectrum in the entire raw file must be deconvoluted. In an effort to maintain consistency between analysis techniques, we used MSConvert to deconvolute RAW files in this study. However, we have also implemented a simple deconvolution algorithm in EncyclopeDIA that can be performed on-the-fly while reading spectra in a narrow I/O buffer. In a DIA data set, at each cycle $(T)$ every MS/MS spectrum $\left(S_{T i}\right)$ comprises fragments from precursors within the precursor isolation window $(i)$. Spectra in consecutive half cycles are overlapped by $50 \%$, such that precursors from the lower $50 \%$ of the window in MS/MS spectrum $S_{T i}$ should also be present in the previous/next half cycles lower offset spectra $\left(S_{(T-1)(i-1)}\right.$ and $\left.S_{(T+1)(i-1)}\right)$ while precursors from the upper $50 \%$ of the window should also be present in the corresponding upper offset spectra $\left(S_{(T-1)(i+1)}\right.$ and $\left.S_{(T+1)(i+1)}\right)$. We divide these windows into two bins and attempt to determine which fragments were derived from precursors in the upper half or the lower half using previous and next half cycles. Fragment ions that are found exclusively on the lower previous/next spectra $\left(S_{(T-1)(i-1)}\right.$ and $\left.S_{(T+1)(i-1)}\right)$ are assigned to the lower bin, while those found exclusively in the upper previous/next spectra $\left(S_{(T-1)(i+1)}\right.$ and $\left.S_{(T+1)(i+1)}\right)$ are assigned to the upper bin. Ions that are found in both sets of spectra are assigned proportionally to each bin where the proportion is set to the summed peak intensity for both spectra, e.g.: $\left(S_{(T-1)(i-1)}+S_{(T+1)(i-1)}\right) /\left(S_{(T-1)(i-1)}+S_{(T+1)(i-1)}+S_{(T-1)(i+1)}+S_{(T+1)(i+1)}\right)$ for the lower bin. Peaks that are found in none of the previous and next overlapping spectra are assumed to be noise. New spectra are built from the deconvoluted peaks in both the lower and upper bins. Since this algorithm only needs to consider three half cycles at a time, deconvolution can happen quickly and in memory, with minimal impact on file reading speeds.

Decoy library entries. A decoy library entry is created for every target library entry. To generate a decoy, first the target peptide sequence (except for digestion enzymespecific termini) is reversed, insuring that the decoy maintains its appearance as a tryptic peptide. Then fragment ions corresponding to amino acids (B/Y for CID, C/Z/Z+1 for ETD) or their expected neutral losses due to modifications (e.g. phosphorylation) are 
calculated for both target and decoy entries. If the precursor charge state is greater than +2 , then +2 fragment ions are also considered. Uncommon neutral loss ions such as Atype ions or loss of water or ammonia are not considered to limit the likelihood of false detections. Fragment ions that correspond to target sequence $\mathrm{m} / \mathrm{zs}$ are transferred to new decoy $\mathrm{m} / \mathrm{zs}$ such that their ion type and index are kept consistent. Delta mass errors in each fragment ion are also maintained to preserve consistency, and all peaks corresponding to the fragment delta mass window are transferred if the library is collected in profile mode. Ions that cannot be assigned to amino acids (such as those corresponding to precursor ions, background noise or interference) are not used by EncyclopeDIA.

Ion weighting estimation. While searching, a unique background is calculated for each precursor isolation window using the prevalence of each fragment ion in the library spectra considered for that window (Supplementary Figure 10). This background helps estimate the interference frequency for any given ion and is used to weight some scores. This distribution is calculated as the frequency that any nominal $\mathrm{m} / \mathrm{z}$ fragment ion (rounded by truncation) appears in entries from the library within the specified precursor window filter. $\mathrm{m} / \mathrm{z}$ frequencies are calculated out to 4000 and a pseudocount is applied to every $\mathrm{m} / \mathrm{z}$ bin to avoid "zero" frequency errors.

Primary scoring and feature scoring functions. The primary score in EncyclopeDIA conceptually draws on the X!Tandem HyperScore. Unlike scoring functions like XCorr in Sequest, the HyperScore does not attempt to account or penalize for ions that do not match the peptide in question, making it ideal for DIA analysis where coeluting peptides are common. The score function is the weighted dot product of the intensities in the acquired spectrum $(I)$ and the library spectrum $(P)$, weighted by a correlation score vector $(C)$, which is discussed in detail in the Chromatogram Library ELIB Generation section. Again, any ions in the library spectrum that do not correspond to the amino acid sequence are not considered in this score. The dot product is multiplied by the factorial of the number of matching ions: 


$$
\text { primary score }=\log _{10}\left(\left(\sum_{i=0}^{n} I_{i} \cdot P_{i} \cdot C_{i}\right) \cdot n !\right)
$$

Sometimes modified peptides (for example, oxidized peptides) are present in the same precursor isolation window as their unmodified forms. Since often these peptides share several fragment ions in common, we require that at least $25 \%$ of the score contribution for modified peptides come from ions that exclusively indicate that modification in cases where any of up to four isotopic peaks from the modified/unmodified peptide pairs fall in the same window.

Several more computationally expensive secondary feature scores (Supplemental Table 5) are calculated once peaks are assigned. Briefly, the scores are divided to cover various classes of features: overall scoring (deltaCN, eValue, logDotProduct, logWeightedDotProduct, xCorrLib, xCorrModel), fragment ion accuracy (sumOfSquaredErrors, weightedSumOfSquaredErrors, numberOfMatchingPeaks, averageAbsFragDeltaMass, averageFragmentDeltaMass), precursor ion accuracy (isotopeDotProduct, averageAbsPPM, averagePPM), and retention time accuracy (deltaRT). The deltaRT score is only used after retention time alignment has been performed. All of these scores are fed to Percolator 3.1 for target/decoy FDR analysis.

Retention time alignment. Accuracy and stability of retention time alignments is critical for EncyclopeDIA. Consequently, we designed a new algorithm that works analogous to how we visualize densities. This approach uses two dimensional kernel density estimates $(K D E)$ that are much less prone to failure as compared to typical line fitting approaches such as LOESS in situations with grossly variable numbers of points and outliers. In this approach each X/Y coordinate is estimated as a symmetrical, twodimensional kernel based on a cosine-based Gaussian approximation. Following Silverman's rule(3) the KDE bandwidth is set to:

$$
\text { bandwidth }=N^{-\frac{1}{6}} \cdot\left(\frac{\operatorname{stdev}(x)+\operatorname{stdev}(y)}{2}\right)
$$


where $\mathrm{N}$ is the number of matched peptides. The kernel's standard deviation is set to the bandwidth (analogous to full width at half max) divided by $2 \sqrt{2 \cdot \ln (2)}$. This distribution is stamped at every $X / Y$ coordinate on a 1000 by 1000 grid mapping from the lowest and highest retention times in both the $X$ and $Y$ dimensions. Once the KDE is calculated, the optimal fit is traced using a ridge walking algorithm that traces the mode of the KDE across retention time (Supplementary Figure 11). In this algorithm the highest point in the KDE is identified and the line is fit in increasing retention time by moving to the highest local grid point to the north (increased sample retention time), east (increased library retention time), or northeast. If north and east are both the highest local point, then the line moves to the northeast. This is performed iteratively until the line is fit across the increasing retention time. Then the same ridge walk is performed in decreasing retention time by moving south, west, or southwest. This approach forces a monotonic line (it can never find a negative retention time change) that follows where the most number of $X / Y$ coordinates lie.

Retention time alignment mixture model. After the alignment is performed, we use the delta retention time data to produce a mixture model to determine outliers. We calculate a Gaussian distribution representing "correct" retention time matches using the median delta retention time as the Gaussian mean and interquartile range divided by 1.35 as the Gaussian standard deviation. We use a unit distribution to represent "incorrect" retention time matches. Starting where the distribution priors are set to 0.5 , we run 10 iterations of a PeptideProphet-like mixture model(4) to fit the two distributions to the delta retention time data using an Expectation Maximization algorithm(5). Peptide matches with posterior error probability estimations that are less than $5 \%$ likely to be in the "correct" retention time distribution are considered outliers.

Retention time alignment across experiments. For each passing peptide, we determine the experiment that produced the best scoring match and set that match aside as a "canonical" peptide representation. We chose the experiment with the most canonical peptides as an anchor and retention time align all of the experiments (and their canonical peptides) to that anchor. Mixture models (described above) for these retention time alignments are calculated and outliers are removed if the local-anchor 
delta retention time is less than $0.1 \%$ likely to fit the mixture model. New retention times for outlier-removed peptides and peptides that were only assigned globally are inferred using the anchor retention time.

FDR filtering peptide and protein detections across experiments. We concatenate peptide feature files from all experiments in a study and run Percolator 3.1 to perform global peptide FDR filtering at 0.01. Using this list of peptides, we generate a parsimonious list of protein groups using a greedy algorithm. Here peptides are assigned to protein groups with the highest protein score:

$$
\operatorname{protein} \operatorname{score}(P)=N-\sum_{p \in P}^{N}\left(P E P_{p}\right)
$$

where the the sum of the peptide $(p)$ posterior error probabilities $\left(P E P_{p}\right)$ is subtracted from the number of peptides $(N)$ assigned to that protein $(P)$. Protein groups are sorted on the lowest $P E P_{p}$ assigned to them(6) and then stringently target/decoy filtered to 0.01 protein FDR.

Automated transition refinement. Fragment ion interference is common when analyzing wide-window data. While fragment ions that show interference may still be useful for detecting peptides, those ions must be screened prior to quantitation to ensure an accurate measurement. We designed a new non-parametric approach to selecting the best ions for quantitation. We first Savizky-Golay smooth(7) the fragment ion chromatograms and then normalize them to have unit integrated intensity. To simplify the smoothing mathematics, we make the assumption that cycle times are consistent within the time frame of a single peak, thus removing the need for interpolation over retention time. After normalization the chromatograms of quantitatively useful ions line up while those of interfered ions will have either higher or lower unit-normalized intensities at different retention times. We calculate the median normalized intensity at each retention time point as an approximation for the peptide peak shape. We then determine peak boundaries by tracing descent of the median peak shape from the maximum normalized intensity on either side of the peak. The 
boundaries are set to the minimum point at which the median peak trace starts increasing for $>2$ consecutive spectra or any point where the trace drops to less than $1 \%$ of the maximum. At that point we calculate a Pearson's correlation coefficient for the similarity between each fragment ion chromatogram with that of the median peak shape between those boundaries. Peaks that match with a correlation coefficient of at least 0.9 are considered quantitative, while those that match with coefficients of at least 0.75 are considered useful for detection purposes.

Fragment ion quantification and background subtraction. We calculate trapezoidal peak areas across Savitsky-Golay smoothed chromatograms. Analogous to Skyline, peak intensities are background subtracted by removing a peak area rectangle with a height equal to the largest intensity of either of the boundary edges. If the area of the rectangle is larger than the area of the peak the intensity is set to zero.

\section{Peptide quantification and transition choice across experiments. Transition}

interference changes on a sample by sample basis. We rank quantitative transitions ( $>0.9$ correlation) based on the sum of their correlation scores across all experiments (effectively counting the number of samples in which they are observed). In addition, for each transition we calculate a global interference score:

$$
\operatorname{interference~score}(t)=\frac{\sum_{s} I_{t, s}\left[C_{t, s}<0.9\right]}{\sum_{s} I_{t, s}\left[C_{t, s}>=0.9\right]}
$$

Which represents the sum of transition $(t)$ intensities $\left(l_{t, s}\right)$ across experiments $(s)$ that show interference $\left(C_{t, s}<0.9\right)$ over those that do not $\left(C_{t, s} \geq 0.9\right)$. Transitions with interference scores $>0.2$ are deemed untrustworthy for quantification and are dropped. Peptide quantities are set to the sum of the top 5 transitions that pass these criteria, where peptides with fewer than 3 quantitative transitions are not carried forward. We require additional stringent criteria for our time course study. Specifically, we required that each peptide be measured in every replicate of at least one time point, and that cross experiment CVs (estimated using quantities from each time point corrected with a linear model) be less than $20 \%$. 
Protein quantification and statistical testing. Protein quantities were calculated as the sum of peptide quantities. We used Extraction of Differential Gene Expression (EDGE) 3.6(8) to statistically test for reproducible changes across the time course study. We performed k-means clustering of proteins that passed an EDGE q-value filter of 0.01 using 5 groups using 1,000 random starting points with 1,000 iterations. We estimated 5 groups by calculating the sum of within squared errors of each $\mathrm{K}$ model from 1 to 15 and estimating the first point where the change in the sum of within squared errors was flat (Supplementary Figure 12).

Gene Ontology enrichment. We performed Gene Ontology enrichment of significantly changing proteins using the online PANTHER Overrepresentation Test(9) (release 20170413) with the Homo sapiens Gene Ontology database (release 2017-10-24) using a background of all proteins consistently detected in our experiments. After removing terms with fewer than 20 proteins (to avoid weakly powered classes) and more than 1,000 proteins (to avoid vague classes), we applied Benjamini-Hochberg FDR correction and filtered enrichment tests to a FDR $<0.05$.

EncyclopeDIA implementation and software/data availability. EncyclopeDIA is implemented in Java 1.8 as both a command line and a stand-alone GUI application. EncyclopeDIA supports the HUPO PSI mzML standard for reading raw MS/MS data, and can construct DLIB DDA-based spectrum libraries from Skyline/Bibliospec BLIB files, NIST MSP files, or HUPO PSI TraML files. Additionally, EncyclopeDIA results can be imported into Skyline(10) to enable further visualization and downstream processing. EncyclopeDIA is heavily optimized and multi-threaded such that searches can be performed on conventional desktop computers with limited RAM and processing power. We have released source code and cross platform (Windows, Mac OS X, Linux) binaries for EncyclopeDIA on Bitbucket at: https://bitbucket.org/searleb/encyclopedia under the open source Apache 2 license. All mass spectrometry mzML and RAW data files are available on the Chorus Project (Supplementary Table 3). 


\section{SUPPLEMENTARY REFERENCES}

1. Frewen BE, Merrihew GE, Wu CC, Noble WS, MacCoss MJ. Analysis of peptide MS/MS spectra from large-scale proteomics experiments using spectrum libraries. Anal Chem. 2006;78:5678-5684.

2. Lawrence RT, Searle BC, Llovet A, Villén J. Plug-and-play analysis of the human phosphoproteome by targeted high-resolution mass spectrometry. Nat Methods. 2016;13:431-434.

3. Silverman BW. Density estimation for statistics and data analysis. CRC press; 1986

4. Keller A, Nesvizhskii Al, Kolker E, Aebersold R. Empirical statistical model to estimate the accuracy of peptide identifications made by MS/MS and database search. Anal Chem. 2002;74:5383-5392.

5. Dempster AP, Laird NM, Rubin DB. Maximum likelihood from incomplete data via the EM algorithm. Journal of the royal statistical society Series B (methodological). 19771-38.

6. The M, MacCoss MJ, Noble WS, Käll L. Fast and Accurate Protein False Discovery Rates on Large-Scale Proteomics Data Sets with Percolator 3.0. J Am Soc Mass Spectrom. 2016;27:1719-1727.

7. Savitzky A, Golay MJE. Smoothing and differentiation of data by simplified least squares procedures. Analytical chemistry. 1964;36:1627-1639.

8. Storey JD, Xiao W, Leek JT, Tompkins RG, Davis RW. Significance analysis of time course microarray experiments. Proc Natl Acad Sci U S A. 2005;102:1283712842.

9. Mi H, Muruganujan A, Casagrande JT, Thomas PD. Large-scale gene function analysis with the PANTHER classification system. Nat Protoc. 2013;8:1551-1566.

10. MacLean B, Tomazela DM, Shulman N et al. Skyline: an open source document editor for creating and analyzing targeted proteomics experiments. Bioinformatics. 2010;26:966-968. 